\title{
Excellent Anti-bacterial Activity of Poly(o-toluidine)-DBSA/ ZnO Nanocomposite
}

\author{
Dalal K. Thbayh*1 Rawnaq A. Talib ${ }^{1} \quad$ E. H. Al - Tememe ${ }^{1,2}$ \\ 1.University of Basra, Polymer Research Canter, Basra, Iraq \\ 2.iversity of Basra, Pharmacy College, Basra, Iraq
}

\begin{abstract}
This work presents a study of the biological application (antibacterial activity) of $\mathrm{ZnO} /$ poly(o-toluidine) (POT) doped with organic acids dodecylbenzene sulfonate acid (DBSA) nanocomposites synthesized by in-situ polymerization of (o-toluidine) monomer in presence of $5 \% \mathrm{ZnO}$. The FTIR spectroscopy confirms the existence of an interaction between POT-DBSA matrix and $\mathrm{ZnO}$ particles. Scanning electron microscopy reveals the nanostructure nature of the obtained composite. The antibacterial activity of POT-DBSA/ZnO nanocomposite and POT-DBSA studied by agar well diffusion method, was found to increase with increasing concentration meanwhile POT/DBSA/ZnO exhibits better antibacterial activity compared to POT/DBSA and POT separately.
\end{abstract}

Keywords: Poly(O-toluidine); Organic acid; $\mathrm{ZnO}$; nanocomposite; antibacterial activity.

DOI: $10.7176 /$ APTA/81-04

Publication date: December $31^{\text {st }} 2019$

\section{Introduction}

Synthesizing and doping poly (o-toluidine) with organic acid such as DBSA (dodecylbenzene sulfonate acid) is useful for various applications. Poly (o-toluidine) (POT) as dopant is one of the most conducting polymer promising to substitute polyaniline (D. Macinnes 1988, Watanabe, Mori et al. 1989, Wei, Focke et al. 1989) due to its good chemical stability with high conductivity, easy polymerization, since polyaniline shows difficult solubility in majority of solvents. Therefore, POT doped with DBSA containing long alkyl chains could be soluble in common solvents like chloroform, xylene, m-cresol and toluene (T. Taka 1994, Athawale, Kulkarni et al. 2002 , Ebrahim, Kashyout et al. 2009). To solve the problem of poor chemical, thermal and mechanical properties of conducting polymers for particular applications, a considerable amount of research work has been devoted to prepare hybrid inorganic/organic composites, which are formed by inserting inorganic particles to the polymer chain structure in order to obtain unique properties (Khan, Khalid et al. 2010, Ansari and Mohammad 2011, Khan, Baig et al. 2011, Khan and Baig 2012, Khan and Baig 2013, Khan and Baig 2013).

Zinc oxide $(\mathrm{ZnO})$ considered as one of the versatile inorganic compound belonging to II-VI group compound, is an n-type semiconductor with a direct wide band gap $(3.37 \mathrm{eV})$ and a large exciton binding energy at room temperature (about $60 \mathrm{meV}$ ) (Sui, Shao et al. 2007).

$\mathrm{ZnO}$-based nanostructured materials represent an important class of semi conductive oxides that can be used in many applications because of its low cost with a diversity of forms, such as nanorods (Park, Kim et al. 2002, Hassan, Hassan et al. 2011), nanofibers, nanoparticles, nanobelts, nanoflowers (Wang, Qian et al. 2004, Izaki, Watanabe et al. 2008) and nanowires (Zhang, Ram et al. 2012). G. Sudhana and co-workers (Sudhana, Thenmozhi et al. 2012) studied the antibacterial activity of POT prepared by chemical oxidation method in the presence / absence of ultrasonic irradiation, it's results shows the antibacterial activity and conductivity of POT prepared in the presence of ultrasonic irradiation is better than its absence. In recent decades, many works have been synthesized of polymer-metallic nanocomposite and studied their antibacterial activities (Khan, Radhakrishnan et al. 2018).

This work involves preparation of composites from inorganic 5\% weight ratios $\mathrm{ZnO}$ nanoparticles and organic conducting polymer of poly (o-toluidine) doped with dodecylbenzene sulfonate (POT-DBSA) by in-situ chemical oxidative polymerization method. The inhibition zone experiment was applied to evaluate the antibacterial activity of POT-DBSA/ZnO nanocomposite and POT-DBSA by using three types of bacteria Escericha Coli and Pseudomones aerogmosa (Gram negative) and Staphlococcus aureus (Gram positive), respectively.

\section{Experimental}

2.1 Synthesis of poly-o-toluidine doped with dodecyl benzene sulfonic acid (POT-DBSA)

The polymerization of the monomer o-toluidine (Fisher scientific company, USA), was initiated by the drop wise addition of the oxidizing agent, (5.8 gm, 1M) ammonium persulphate (APS) (NH4)2S2O8 (Sigma Aldrich, USA). 5.4 gm organic acid Dodecylbenzene sulfonate (DBSA) (Sigma Aldrich, USA) was used for doping POT. The POT to ammonium persulphate mixing in similar volume ratios is achieved by continuous stirring using a magnetic stirrer for $24 \mathrm{~h}$. A green colored gel was obtained (Ahmad, Riaz et al. 2012, Khan and Baig 2013, Thbayh DK 2018) which was filtered, and repeatedly washed with distilled water to remove the excess of acid and impurities then finally dried at $70 \mathrm{oC}$ for $6 \mathrm{~h}$ in an oven. The final product was a green powder (POT-DBSA). 


\subsection{Synthesis of POT-DBSA/ ZnO nanocomposite}

POT-DBSA/ZnO nanocomposite was prepared by in situ oxidative polymerization of o-toluidine in the presence of $\mathrm{ZnO}$ (Sigma Aldrich, USA) nanoparticles (NPs). 5wt\% of ZnO NPs were poured into solution of o-toluidineDBSA and polymerization was affected by the addition of solution containing oxidant ammonium persulphate prepared in $1 \mathrm{M} \mathrm{HCl}$. The addition of oxidant solution led to the polymerization of adsorbed o-toluidine on $\mathrm{ZnO}$ NPs resulting in the appearance of light greenish black precipitate colored solution which was kept under continuous stirring for $24 \mathrm{~h}$. The reaction mixture was then filtered, washed with double distilled water to remove excess acid. The nanocomposite thus prepared was dried at $70 \mathrm{oC}$ for $6 \mathrm{~h}$ in an oven, converted into powders and stored in desiccator for further investigations.

\subsection{Antibacterial activity}

The Muller Hinton agar was used for the growth of Escherichia coli and Pseudomonas aerogmosa (Gram negative) and staphylococcus aurous (Gram positive) bacteria to study the antibacterial activity of POT-DBSA/ ZnO nanocomposite and POT-DBSA, by using the diffusion in the agar method. The bacterial strain was cultured on the nutrient agar broth to produce a new colony of age $24 \mathrm{~h}$, then to obtain a bacterial growth one new colony from the growth was inoculated on a $5 \mathrm{ml}$ of nutrient broth and was incubated in $37 \mathrm{C}$ for $6 \mathrm{~h}$. A $0.1 \mathrm{ml}$ from the growth was separated on the Muller-Hinton agar. The inoculated agar surface was poured in $4 \mathrm{~mm}$ diameter for every pore. The pores of cultured media were filled by 100, 200, 300, 400 and $500 \mu \mathrm{g} / \mathrm{ml}$ of POT-DBSA/ZnO nanocomposite and POT-DBSA, the controlled solution was $10 \mathrm{ml}$ of Dimethyl- sulpha oxide. Then the dishes were kept in the incubator at $37^{\circ} \mathrm{C}$ for a period of $18-24 \mathrm{~h}$. The inhibition zone diameters in mm were measured around the pores.

\subsection{Characterizations}

The morphology of nano composites films was investigated by field emission scanning electron microscopy (FESEM) using FEINova Nano SEM 450. Fourier transform infrared (FT-IR) spectra were recorded on FT-IR 4200 Jasco.

\section{Results and Discussion}

\subsection{FTIR spectroscopy analysis}

The FT-IR spectrum of the POT-DBSA/ ( $5 \mathrm{wt} \% \mathrm{ZnO}$ ) nanocomposite is shown in Fig 1 . The characteristic bands of POT-DBSA are as follows: the peak at $1604 \mathrm{~cm}-1$ is ascribed to the stretching vibration of quinoid rings; the peak at $1406 \mathrm{~cm}-1$ depicts the presence of benzenoid ring units (Kulkarni, Viswanath et al. 2004, Ebrahim, Gad et al. 2010); the peak at $1170 \mathrm{~cm}-1$ is assigned to a vibration band of the dopant ion, which is formed during protonation of POT with DBSA (Rao, Sathyanarayana et al. 2002, Kulkarni, Viswanath et al. 2004); the peak at $3121 \mathrm{~cm}^{-1}$ is attributed to the free N-H stretching vibration (Kulkarni, Viswanath et al. 2004), the peak at 1004 cm-1 corresponds to the C-H in plan bending vibration (Kulkarni, Viswanath et al. 2004); while the peaks at 682 and $582 \mathrm{~cm}-1$ are associated to C-H out of plane bending vibration (Kulkarni, Viswanath et al. 2004) and C-N-C bonding mode of aromatic ring, respectively. The characteristic peak at $436 \mathrm{~cm}-1$ belongs to the $\mathrm{Zn}-\mathrm{O}$ stretching mode (Hamedani and Farzaneh 2006, Kakazey, Vlasova et al. 2007, Geetha and Thilagavathi 2010, Zhao, Cai et al. 2011, Nosrati, Olad et al. 2012). The frequency data obtained, and their assignments are presented in Table 1.

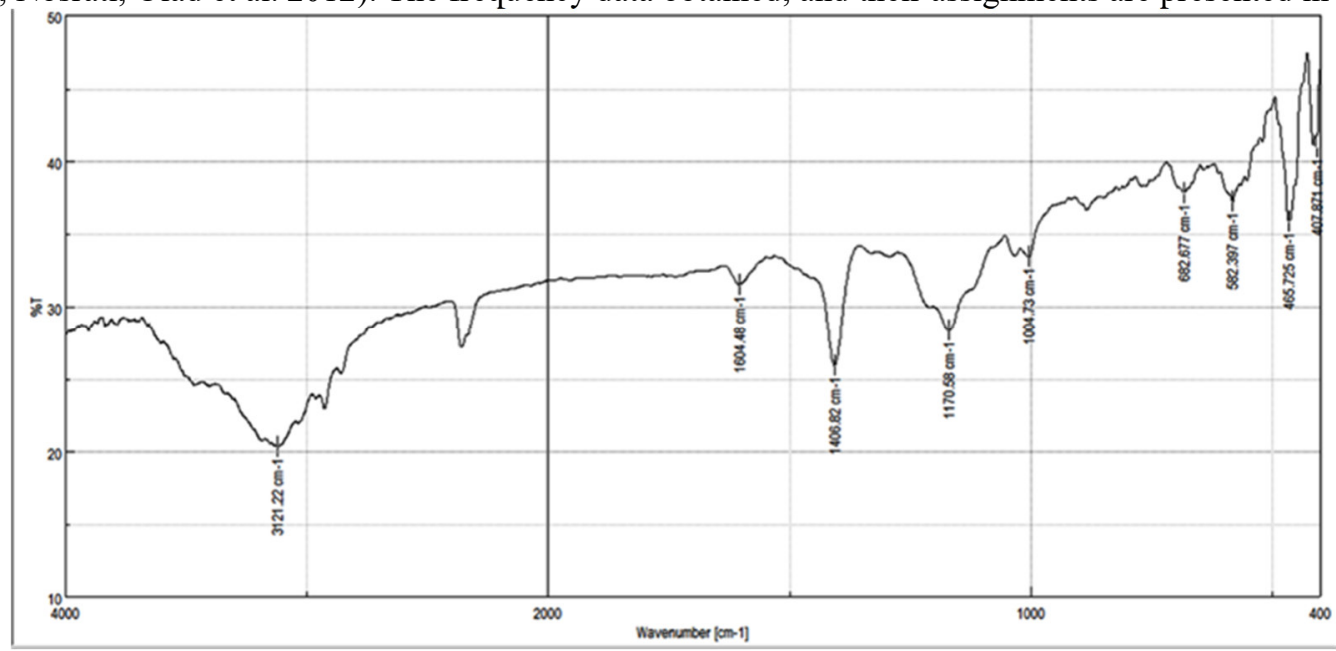

Figure 1. FTIR spectrum of POT-DBSA/5\% ZnO nanocomposite. 
Table 1. Characteristic frequencies of in-situ chemically synthesized modes of the POT-DBSA $/ 5 \% \mathrm{ZnO}$ nanocomposite.

\begin{tabular}{|c|c|}
\hline Assignment & $\begin{array}{c}\text { Wave number } \mathbf{( c m}^{-1} \mathbf{)} \\
\text { POT-DBSA/5\%(n- }\end{array}$ \\
\hline Zn-O & 407.8 \\
& 465.7 \\
C-N-C bonding mode of aromatic ring & 582.3 \\
C-H out of plane bonding in benzenoid ring & 682.6 \\
C -H in plane bonding & 1004.7 \\
Vibration band of the dopant ion & 1170.5 \\
C = C stretching mode of the benzenoid rings & 1406.8 \\
C=C stretching mode of the quinoid rings & 1604.4 \\
H-N stretching vibration & 3121.2 \\
\hline
\end{tabular}

\subsection{FESEM and EDX analysis}

Figure2 shows the basic chemical structure of POT, DBSA, and ZnO. The EDX spectrum of POT-DBSA $/ 5 \% \mathrm{ZnO}$ shown in the Table 2, reveals the presence of $\mathrm{Zn}, \mathrm{O}, \mathrm{S}$, and C elements. It can be deduced that the presence of $\mathrm{Zn}$ element in composite results from the interaction of POT-DBSA with $\mathrm{ZnO}$ nanoparticles. The SEM image of nanocomposite film is shown in Fig 3. It can be observed that the crystals of ZnO NPs are fully covered by POT and the composite shows a globular-type nanostructure consisting of small globules and pores. This result indicates that $\mathrm{ZnO} \mathrm{NPs}$ are encapsulated into the POT matrix hence can significantly improve the compactness of the polymer. The average diameter of POT-DBSA/ZnO grains is found to be $37 \mathrm{~nm}$.

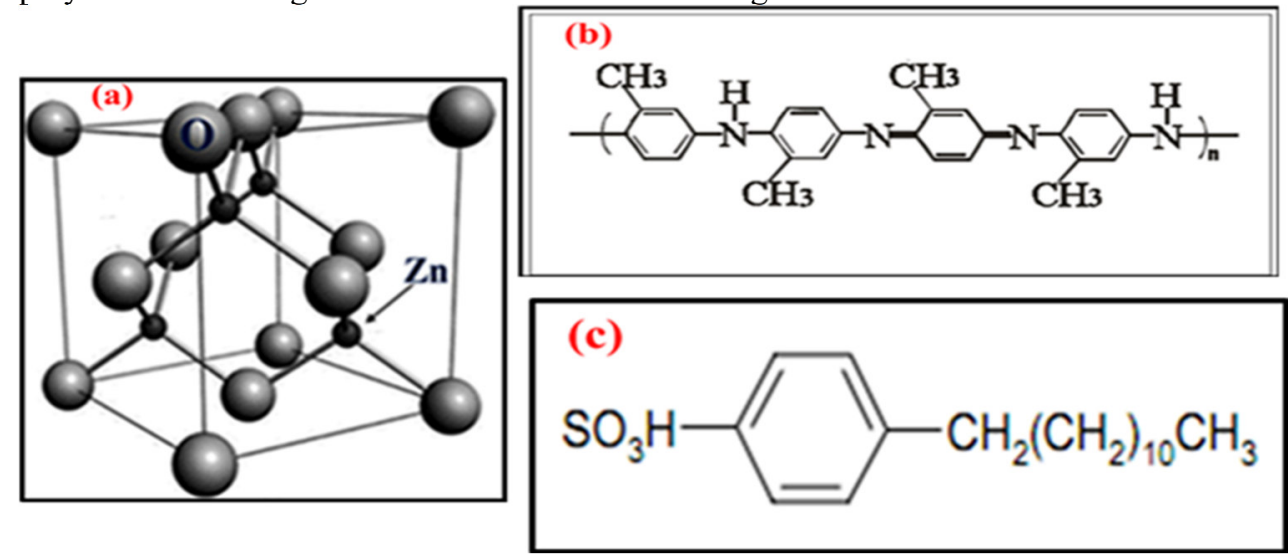

Figure 2. The basic chemical structure of (a) ZnO, (b) POT, and (c) DBSA (Vaseem, Umar et al. 2010, Jagadish and Pearton 2011). 
Table 2. EDX data of POT-DBSA/ 5\% ZnO

\begin{tabular}{|lll|}
\hline Elt & W\% & A\% \\
\hline C & 62.02 & 75.49 \\
O & 17.77 & 16.23 \\
Al & 1.11 & 0.60 \\
S & 14.56 & 6.64 \\
Fe & 0.68 & 0.18 \\
Zn & 3.87 & 0.86 \\
& 100.00 & 100.00 \\
\hline
\end{tabular}

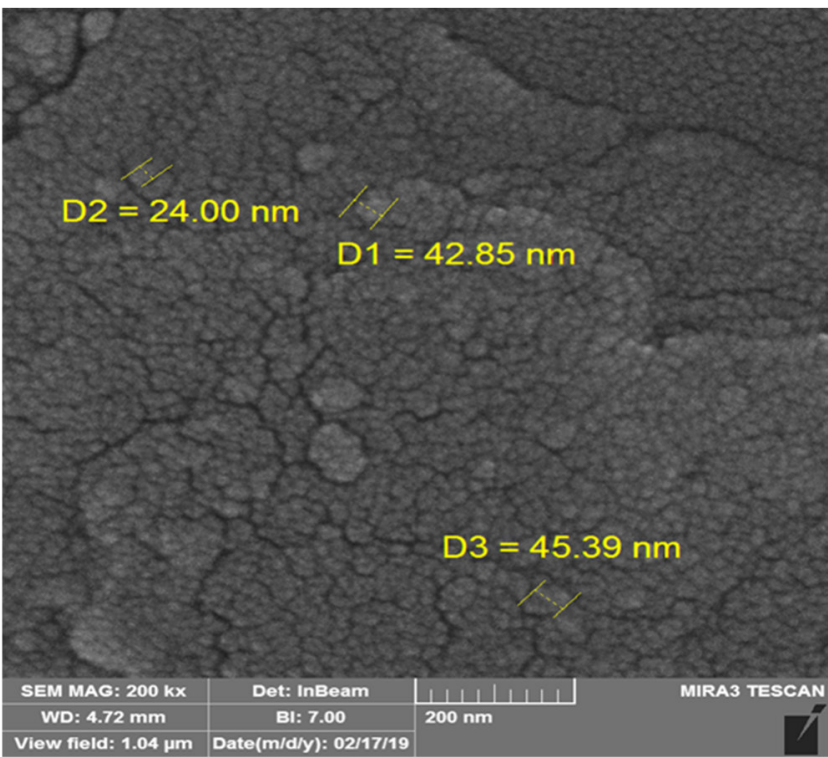

Figure 3. FESEM images of POT-DBSA/5\% ZnO nanocomposite.

3.3 Determination the inhibition zone diameter

The inhibition zone diameters against Staphylococcus aurous, Escherichia coli and Pseudomonas aerogmosa bacteriaby POT-DBSA/ZnO nanocomposite and POT-DBSA which were compared with POT taken separately and with standard drug. There are four types of antibacterial activities (R. Kavitha 2011): (i) the inhibition zone diameter $>>12 \mathrm{~mm}$ resulting in high sensitive bacteria; (ii) moderate sensitive bacteria for the inhibition zone diameter in the range 9-12 $\mathrm{mm}$; (iii) if the inhibition zone diameter is in the range 6-9 $\mathrm{mm}$, then the sensitivity of bacteria is less; and (iv) bacteria becomes resistant if then inhibition zone diameter is $<6 \mathrm{~mm}$. From Table 3 and photographic pictures shown in Figure 4, it can be indicated that the inhibition zone diameter is highest for POTDBSA/ZnO nanocomposite and POT-DBSA; and that the inhibition zone diameter increases as the concentration of the nanocomposite increases.

From Table 3 the following points can be highlighted:

1. POT-DBSA/ZnO nanocomposite exhibits a better antibacterial activity than POT-DBSA, POT and Oxytetracyclin drug.

2. The inhibition zone diameter against Pseudomonas aerogmosaand Escherichia coli (gram negative) is relatively higher than against Staphylococcus aureus (gram negative) due to the difference in the cell wall between gram positive and gram-negative bacteria. The increase in the inhibition zone diameter may be due to the presence of $\mathrm{ZnO}$ NPs in POT-DBSA/ZnO nanocomposite because the antibacterial activity has been reported to be size dependent (Boomi, Prabu et al. 2014), then the bacterial death due to electromagnetic 
attraction between the bacteria (negative charge) and $\mathrm{ZnO}$ nanocomposite (positive charge) (A. Thomas1 2014). In addition, the effect of DBSA in POT-DBSA may destruct bacterial enzymes by coordinating to the electron donating groups, thus DBSA causes deformities in bacterial cell wall to render them leaky (Shakir, Khan et al. 2014).

Table 3. The inhibition zone diameter of three types of bacteria by POT-DBSA/ZnO nanocomposite, POT-DBSA, POT and drug.

\begin{tabular}{|c|c|c|c|c|c|c|c|c|c|}
\hline \multicolumn{5}{|c|}{ POT-DBSA/ZnO nanocomposite } & \multicolumn{5}{|c|}{ POT-DBSA } \\
\hline & Conc. $\mu \mathrm{g} / \mathrm{ml}$ & Staph & E. coli & Psed. & & Conc. $\mu \mathrm{g} / \mathrm{ml}$ & Staph. & E. coli & Psed. \\
\hline 5 & 100 & 10 & 18 & 18 & 10 & 100 & 6 & 12 & 16 \\
\hline 4 & 200 & 12 & 20 & 20 & 9 & 200 & 8 & 16 & 18 \\
\hline 3 & 300 & 16 & 22 & 21 & 8 & 300 & 9 & 20 & 19 \\
\hline 2 & 400 & 18 & 24 & 22 & 7 & 400 & 10 & 22 & 20 \\
\hline 1 & 500 & 20 & 28 & 25 & 6 & 500 & 12 & 25 & 22 \\
\hline \multicolumn{2}{|r|}{ POT } & 10 & 17 & 10 & & & & & \\
\hline \multicolumn{2}{|c|}{ Oxytetracyclin } & 16 & 24 & 22 & & & & & \\
\hline
\end{tabular}

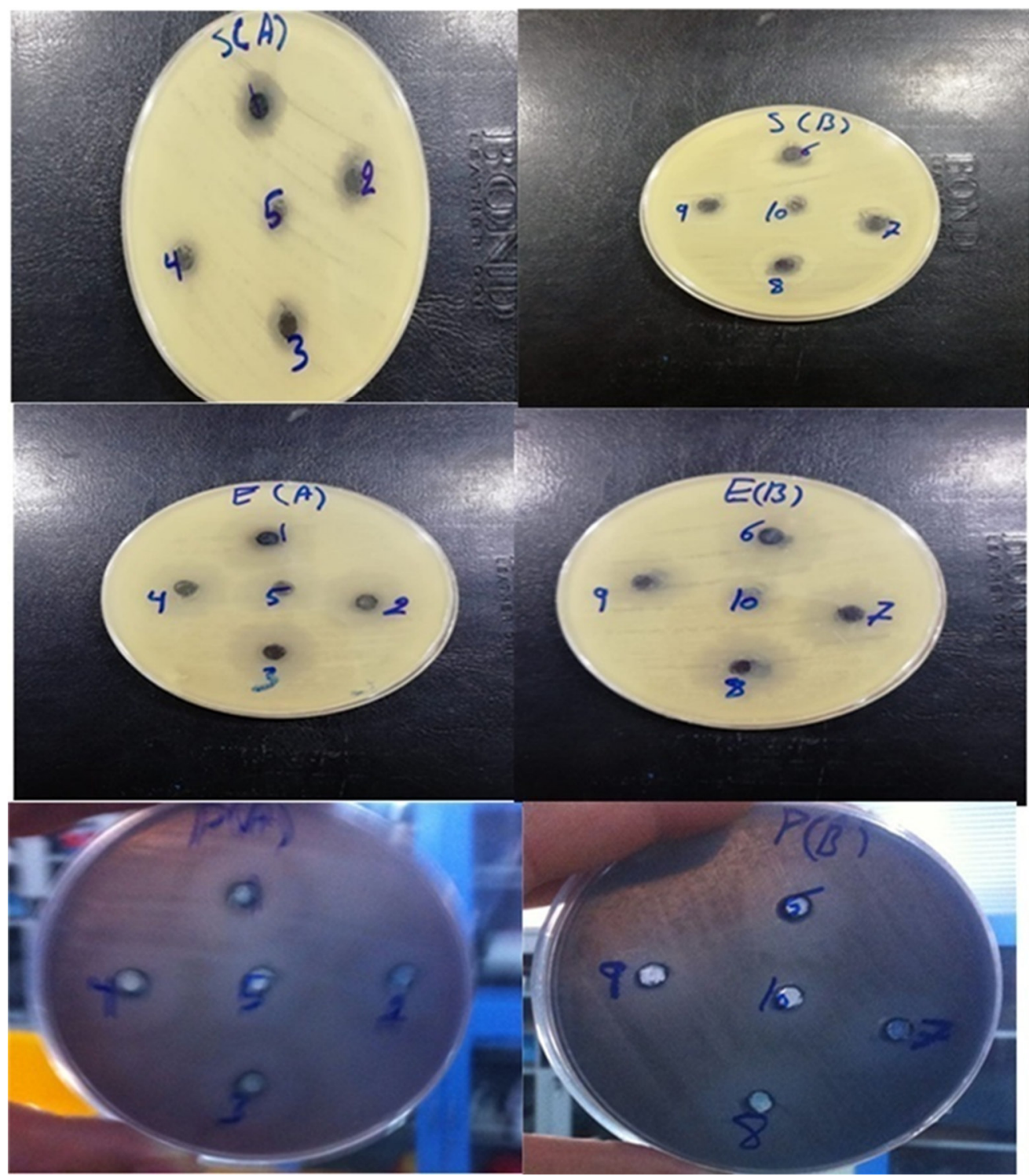

Figure4. Photographic the inhibition zone diameter of (A) POT-DBSA/ZnO nanocomposite and (B) POT-DBSA against (S) Staphylococcus aurous bacteria, (E) Escherichia coli bacteria and (P) Pseudomonas aerogmosa bacteria. 


\section{Conclusion}

POT- DBSA $/ 5 \% \mathrm{ZnO}$ were successfully prepared using in-situ polymerization of (o-toluidine) monomer in the presence of 5\% weight ratio of ZnO NPs. Characteristic bands of $\mathrm{ZnO}$ and POT-DBSA were identified by FTIR and confirming the interaction between $\mathrm{ZnO}$ and polymer matrix. FESEM observations reveal rock salt structure embedded within the polymeric structure, signifying that ZnO NPs are fully covered by POT, with an average diameter of POT-DBSA/ZnO grains around $37 \mathrm{~nm}$. The comparative study of biological application (antibacterial activities) of POT, POT-DBSA, POT-DBSA/ZnO nanocomposite and oxytetraacyclin drug have been investigated. The results confirm that POT-DBSA/ZnO nanocomposite exhibits enhanced antibacterial activity against E.coli and Pseud. aerogmosa Gram negative and Staph. aureus Gram positive bacteria, as well that the inhibition growth of bacteria increases when POT-DBSA are coordinated with ZnO NPs. The antibacterial properties could make POT-DBSA/ZnO nanocomposite potentially useful as novel drug, thereby future in-vivo measurements are underway.

\section{References}

A. Thomas1, M. S. R. a. J. V., Int. (2014). Journal Bioassays 3: 3106-3110.

Ahmad, S., U. Riaz, M. Kashif and M. S. Khan (2012). "Development of Nanostructured Poly (o-toluidine) Reinforced Organic-Inorganic Hybrid Composites." Journal of Inorganic and Organometallic Polymers and Materials 22(3): 662-670.

Ansari, M. O. and F. Mohammad (2011). "Thermal stability, electrical conductivity and ammonia sensing studies on p-toluenesulfonic acid doped polyaniline: titanium dioxide (pTSA/Pani: TiO2) nanocomposites." Sensors and Actuators B: Chemical 157(1): 122-129.

Athawale, A. A., M. V. Kulkarni and V. V. Chabukswar (2002). "Studies on chemically synthesized soluble acrylic acid doped polyaniline." Materials Chemistry and Physics 73(1): 106-110.

Boomi, P., H. G. Prabu and J. Mathiyarasu (2014). "Synthesis, characterization and antibacterial activity of polyaniline/Pt-Pd nanocomposite." European journal of medicinal chemistry 72: 18-25.

D. Macinnes, B. L. F. (1988). Synth. Met 25 142-235.

Ebrahim, S., A.-H. Kashyout and M. Soliman (2009). "Ac and Dc conductivities of polyaniline/poly vinyl formal blend films." Current Applied Physics 9(2): 448-454.

Ebrahim, S. M., A. Gad and A. Morsy (2010). "Highly crystalline and soluble dodecylbenzene sulfonic acid doped poly (o-toluidine)." Synthetic Metals 160(23-24): 2658-2663.

Geetha, D. and T. Thilagavathi (2010). "Hydrothermal synthesis of nano ZnO structures from CTAB." Digest Journal of Nanomaterials and Biostructures 5(1): 297-301.

Hamedani, N. F. and F. Farzaneh (2006). "Synthesis of ZnO nanocrystals with hexagonal (Wurtzite) structure in water using microwave irradiation." Journal of Sciences, Islamic Republic of Iran 17(3): 231-234.

Hassan, J., Z. Hassan and H. Abu-Hassan (2011). "High-quality vertically aligned ZnO nanorods synthesized by microwave-assisted CBD with ZnO-PVA complex seed layer on Si substrates." Journal of Alloys and Compounds 509(23): 6711-6719.

Izaki, M., M. Watanabe, H. Aritomo, I. Yamaguchi, S. Asahina, T. Shinagawa, M. Chigane, M. Inaba and A. Tasaka (2008). "Zinc oxide nano-cauliflower array with room temperature ultraviolet light emission." Crystal Growth and Design 8(4): 1418-1421.

Jagadish, C. and S. J. Pearton (2011). Zinc oxide bulk, thin films and nanostructures: processing, properties, and applications, Elsevier.

Kakazey, M., M. Vlasova, M. Dominguez-Patiño, I. Leon and M. Ristic (2007). "Reactionary processes during mechanical treatment of mixtures of $\mathrm{ZnO}$ and $\mathrm{MnO}$ 2. I. Formation of defects and solid solution." Journal of materials science 42(17): 7116-7122.

Khan, A. A. and U. Baig (2012). "Electrically conductive membrane of polyaniline-titanium (IV) phosphate cation exchange nanocomposite: Applicable for detection of $\mathrm{Pb}$ (II) using its ion-selective electrode." Journal of Industrial and Engineering Chemistry 18(6): 1937-1944.

Khan, A. A. and U. Baig (2013). "Electrical conductivity and humidity sensing studies on synthetic organicinorganic Poly-o-toluidine-titanium (IV) phosphate cation exchange nanocomposite." Solid State Sciences 15: 47-52.

Khan, A. A., U. Baig and M. Khalid (2011). "Ammonia vapor sensing properties of polyaniline-titanium (IV) phosphate cation exchange nanocomposite." Journal of hazardous materials 186(2-3): 2037-2042.

Khan, A. A. and U. J. S. S. S. Baig (2013). "Electrical conductivity and humidity sensing studies on synthetic organic-inorganic Poly-o-toluidine-titanium (IV) phosphate cation exchange nanocomposite." 15: 47-52.

Khan, A. A., M. Khalid and U. Baig (2010). "Synthesis and characterization of polyaniline-titanium (IV) phosphate cation exchange composite: methanol sensor and isothermal stability in terms of DC electrical conductivity." Reactive and functional polymers 70(10): 849-855.

Khan, J. S., A. Radhakrishnan and B. Beena (2018). "Indian Journal of Advances in Chemical Science." Indian 
Journal of Advances in Chemical Science 6(2): 71-76.

Kulkarni, M. V., A. K. Viswanath, R. Marimuthu and U. Mulik (2004). "Investigation of effect of protonic acid media on the optical and thermal properties of chemically synthesized poly (o-toluidine)." Journal of Materials Science: Materials in Electronics 15(12): 781-785.

Nosrati, R., A. Olad and R. Maramifar (2012). "Degradation of ampicillin antibiotic in aqueous solution by $\mathrm{ZnO}$ /polyaniline nanocomposite as photocatalyst under sunlight irradiation." Environmental Science and Pollution Research 19(6): 2291-2299.

Park, W. I., D. H. Kim, S.-W. Jung and G.-C. Yi (2002). "Metalorganic vapor-phase epitaxial growth of vertically well-aligned ZnO nanorods." Applied Physics Letters 80(22): 4232-4234.

R. Kavitha, P. K., T. Deepa,R. Elamathi, S. Sridhar, J. Suresh Kumar. (2011). Journal of Chemical and Pharmaceutical Research 3(6): 115-121.

Rao, P. S., D. Sathyanarayana and S. Palaniappan (2002). "Polymerization of aniline in an organic peroxide system by the inverted emulsion process." Macromolecules 35(13): 4988-4996.

Shakir, M., M. S. Khan, S. I. Al-Resayes, A. A. Khan and U. Baig (2014). "Electrical conductivity, isothermal stability, and ammonia-sensing performance of newly synthesized and characterized organic-inorganic polycarbazole-titanium dioxide nanocomposite." Industrial \& Engineering Chemistry Research 53(19): 8035-8044.

Sudhana, G., G. Thenmozhi, D. Jayakumar and R. Santhi (2012). "Synthesis, characterization and biological applications of conducting poly o-toludine in the absence and in the presence of ultrasonic irradiation." J. Chem. Pharm. Res 4: 491.

Sui, X., C. Shao and Y. Liu (2007). "Photoluminescence of polyethylene oxide-ZnO composite electrospun fibers." Polymer 48(6): 1459-1463.

T. Taka, J. L., J.K. Levon (1994). Solid State Commun 92: 296-393.

Thbayh DK, Z. K. (2018). "Optical Properties of Conducting Polymer Poly (O-Toluidine)-DBSA Blended with Polyethylene Oxide." Ukrainian Journal of Physics 63(3):

Vaseem, M., A. Umar and Y.-B. Hahn (2010). "ZnO nanoparticles: growth, properties, and applications." Metal oxide nanostructures and their applications 5: 1-36.

Wang, Z., X.-f. Qian, J. Yin and Z.-k. Zhu (2004). "Large-scale fabrication of tower-like, flower-like, and tubelike $\mathrm{ZnO}$ arrays by a simple chemical solution route." Langmuir 20(8): 3441-3448.

Watanabe, A., K. Mori, A. Iwabuchi, Y. Iwasaki, Y. Nakamura and O. Ito (1989). "Electrochemical polymerization of aniline and N-alkylanilines." Macromolecules 22(9): 3521-3525.

Wei, Y., W. W. Focke, G. E. Wnek, A. Ray and A. G. MacDiarmid (1989). "Synthesis and electrochemistry of alkyl ring-substituted polyanilines." The Journal of Physical Chemistry 93(1): 495-499.

Zhang, Y., M. K. Ram, E. K. Stefanakos and D. Y. Goswami (2012). "Synthesis, characterization, and applications of $\mathrm{ZnO}$ nanowires." Journal of Nanomaterials 2012: 20.

Zhao, Y.-P., Z.-S. Cai, Z.-Y. Zhou and X.-L. Fu (2011). "Fabrication of conductive network formed by polyaniline-ZnO composite on fabric surfaces." Thin Solid Films 519(18): 5887-5891. 